\title{
Enoyl-CoA Hydratase Domain-Containing Protein 3, Mitochondrial
}

National Cancer Institute

\section{Source}

National Cancer Institute. Enoyl-CoA Hydratase Domain-Containing Protein 3,

Mitochondrial. NCI Thesaurus. Code C162361.

Enoyl-CoA hydratase domain-containing protein 3, mitochondrial (303 aa, $\sim 33 \mathrm{kDa}$ ) is encoded by the human ECHDC3 gene. This protein may play a role in the metabolism of fatty acids. 